\title{
Applications of Hilbert Transform Theory to Numerical Quadrature*
}

\author{
By W. E. Smith** and J. N. Lyness
}

\begin{abstract}
Some finite integrals are difficult to evaluate numerically because the integrand has a high peak or contains a rapidly oscillating function as a factor. If the integrand is an analytic function Cauchy's theorem may be applied to replace the integral by a contour integral, the path being chosen to avoid singularities of the integrand, together with a possible residue contribution. If the integrand has branch singularities in the complex plane close to the interval of integration, the direct application of Cauchy's theorem is not practical. In this paper we show how the theory of Hilbert transforms may be applied to replace the integrand by a different complex valued function whose real part coincides with the integrand on the real line, but which has no singularities in the upper half plane. Using these transformations, integrands whose difficult behavior arises from a factor whose Hilbert transform is known analytically may be treated by carrying out a contour integral of a different function and taking the real part of the result. It is shown by means of examples that such a procedure may result in significant savings in terms of computational effort.
\end{abstract}

1. Introduction. In this paper we shall be in general considering the calculation of a definite integral having finite limits. We take the integration interval to be $[-1,1]$ as a matter of convenience. The integrand is a real function $f(x)$ which is analytic on the integration interval. In cases where no confusion is likely to arise, we refer to the analytic continuation of $f(x)$ into the complex plane as $f(z)$.

It will be seen that the condition that $f(x)$ be real when $x$ is real plays a basic role in the subsequent theory. The theory may still be applied to complex-valued functions if both the real part and the imaginary part of the function are independently real analytic functions; but each part has to be considered separately.

In the text we shall give several numerical examples. For comparison purposes we have used the Modified Adaptive Simpson method (McKeeman [8]) and the Romberg Integration technique (Bauer, Rutishauser and Stiefel [3]) using a practical convergence criterion suggested by Havie [6]. Both routines require a tolerance $\epsilon$ and produce a numerical result for which this accuracy is claimed. However, in practice, the accuracy is usually far higher than the required tolerance $\epsilon$, and so the number of function evaluations used is larger than is in fact necessary. We refer to these routines by the initials (ASM) and ( $\mathrm{R}$ ) respectively.

As an introductory example, we suppose that the value of

Received March 21, 1968, revised August 23, 1968.

* Work performed under the auspices of the U. S. Atomic Energy Commission.

** On leave of absence from the University of New South Wales, Kensington, N. S. W., Australia. 


$$
\int_{-1}^{1} \frac{e^{x}}{x^{2}+a^{2}} d x, \quad a=0.01,
$$

is required to a prescribed accuracy $\epsilon=10^{-6}$. The direct application of standard quadrature routines produces the result 313.172056 only after a large number of function evaluations has been made. For example, the Modified Adaptive Simpson Routine requires 2779 and Romberg Integration requires 4097 . The reason for these large numbers of function evaluations is that the integrand has a high peak at $x=0 ; f(0)=10,000$ while its value near the end points is near unity; $f( \pm 1)=$ $e^{ \pm 1} / 1.0001$. Thus

A simple way of avoiding this difficulty is to apply Cauchy's Residue Theorem.

$$
\int_{-1}^{1} \frac{e^{x}}{x^{2}+a^{2}} d x=\int_{c} \frac{e^{z}}{z^{2}+a^{2}} d z+2 \pi i R
$$

where $C$ is some contour connecting -1 to +1 lying in the upper half-plane, for example the semicircle

$$
C:|z|=1, \quad 0 \leqq \arg z \leqq \pi,
$$

and

$$
R=e^{i a} / 2 i a
$$

is the residue of the integrand at its only pole (at $z=i a$ ) within the region $R$ bounded by the axis and the contour $C$.

For the purpose of numerical evaluation it is necessary to parameterise the curve $C$ along which the contour integral is required. We may set $z=-\exp (-i \pi t)$; the parameter $\pi t$ is then the arc length in the direction of integration. This leads to

$$
\int_{-1}^{1} \frac{e^{x}}{x^{2}+a^{2}} d x=\pi i \int_{0}^{1} \frac{e^{-\exp (-\pi i t)} e^{-\pi i t}}{e^{-2 \pi i t}+a^{2}} d t+\frac{\pi e^{i a}}{a} .
$$

It is also convenient to take advantage of the fact that the left-hand side of (1.5) is clearly real. Consequently we need only calculate the real part of the right-hand side of (1.5) which may be written in the form

$$
\int_{-1}^{1} \frac{e^{x}}{x^{2}+a^{2}} d x=\int_{0}^{1} \operatorname{Re}\left\{\frac{\pi i e^{-\exp (-\pi i t)} e^{-\pi i t}}{\left(e^{-\pi i t}\right)^{2}+a^{2}}\right\} d t+\operatorname{Re}\left\{\frac{\pi e^{i a}}{a}\right\} .
$$

The second term on the right has the value 314.143558. The integral may be calculated to the accuracy $\epsilon=10^{-6}$ using any standard quadrature routine. Its value is -0.971501 and this calculation requires $151(\mathrm{ASM})$ or $33(\mathrm{R})$ function evaluations. These numbers are relatively small partly because of the small magnitude of the integrand (of order unity) but mainly because the integrand is a smoothly varying function of $t$ not having high peaks. However, in making any comparison it should be remembered that the integrand on the right in (1.6) is more difficult to calculate at any point than the integrand on the left.

The foregoing rather trivial example follows a familiar method suggested by Abramowitz [1]. It is included here to provide the background against which a wider theory given below may be described. However, even this simple example does 
indicate the nature of the results to be obtained and their relative advantages and disadvantages. Briefly, a small amount of analytical work is required. In return for this we may be able to exchange a large number of real function evaluations for a small number of complex function evaluations.

It is necessary at this stage to indicate more clearly the type of integrand for which these transformations are likely to be useful. These are integrands of the form

$$
f(x)=w(x) c(x)
$$

where $w(x)$ and $c(x)$ have the following broad characteristics. $c(x)$ the numerically critical factor is an analytically simple function whose properties are well known, but which is responsible for making the numerical integration difficult. Usually if $w(x)=1$, the integral could be evaluated analytically. For example, the functions

$$
\frac{1}{\left(x^{2}+a^{2}\right)^{3 / 2}}, \frac{1}{\sin n x-1.01}, \quad \cos n x, \quad a=0.01, \quad n=20,
$$

occurring as a factor in an integrand cause considerable difficulty and, though simple in structure, are critical here. On the other hand, the same factors with $a=20$ and $n=0.01$ present no difficulty and would not be considered critical. $w(x)$ is a function which has the opposite characteristics. It may be quite complicated in structure, for example

$$
w(x)=\exp \left[-0.1 x^{3}\right]\left(x^{2}+36\right)^{1 / 2} \ln \left((x+20)^{1 / 3}+(x+15)^{1 / 3}\right),
$$

and consequently rule out entirely any analytic solution. However, it is smoothly varying in the interval of integration and, if $c(x)=1$, the integral could be evaluated numerically with little difficulty.

In the example given above the function $1 /\left(x^{2}+a^{2}\right)$ is a critical factor. If the only singularities of the critical factor are poles, all that is necessary is to choose a contour which avoids these poles and to take into account the residues of the poles lying between the chosen contour and the real axis. We deal with this problem in a later section. However, this simple procedure does not work if the function has singularities other than poles. To illustrate this we consider the calculation of the integral

$$
\int_{-1}^{1} \frac{e^{x}}{\left(x^{2}+a^{2}\right)^{1 / 2}} d x, \quad a=0.01
$$

to an accuracy $\epsilon=10^{-6}$. The critical factor

$$
c(x)=\left(x^{2}+a^{2}\right)^{-1 / 2}
$$

has branch point singularities at $z= \pm i a$. Thus any contour is constrained to pass: between these points and consequently passes close to them. This rules out the direct transformation described in the previous example.

In this case we proceed in the following manner. We introduce the function

$$
s(x)=\frac{2}{\pi\left(x^{2}+a^{2}\right)^{1 / 2}}\{\operatorname{arcsinh}(x / a)\}
$$

(this is in fact the Hilbert transform of $-c(x)$ ) and we define the complex function 


$$
\phi(z)=c(z)+i s(z) .
$$

This function has the following properties:

(i) $\operatorname{Re}(\phi(z))=c(z), z$ real,

(ii) $\phi(z)$ has no singularity in the upper half plane, $\operatorname{Im}(z) \geqq 0$.

In view of these properties, we may write

$$
\int_{-1}^{1} w(x) c(x) d x=\operatorname{Re}\left(\int_{-1}^{1} w(x) \phi(x) d x\right)=\operatorname{Re}\left(\int_{c} w(z) \Phi(z) d z\right)
$$

where $C$ is any contour*** in the upper half-plane connecting -1 to +1 . Since $\Phi(z)$ like $c(z)$ and $s(z)$ has a branch point singularity at $z=-i a$, we choose a contour which comfortably avoids this singularity, such as the semicircle used previously:

$$
C:|z|=1, \quad 0 \leqq \arg z \leqq \pi .
$$

The numerical results are of the same nature as in the previous example. The evaluation of (1.10) directly requires $991(\mathrm{ASM})$ or $2049(\mathrm{R})$ function evaluations of $e^{x} /\left(x^{2}+a^{2}\right)^{1 / 2}(x$ real). The evaluation of $(1.16)$ requires $65(\mathrm{R})$ or $295(\mathrm{ASM})$ evaluations of $e^{z}(1+(2 i / \pi)$ arc $\sinh (z / a)) /\left(z^{2}+a^{2}\right)^{1 / 2}$ at complex points $z$ lying on the unit circle.

The key to this procedure is the construction of the function $\phi(z)$ which satisfies (i) and (ii). In fact the unfamiliar reader may be surprised that the function

$$
\phi(z)=\frac{1}{\left(z^{2}+a^{2}\right)^{1 / 2}}\left\{1+\frac{2 i}{\pi} \operatorname{arcsinh}(z / a)\right\}
$$

has no branch point singularity at $z=+i a$ in spite of the fact that both $\left(z^{2}+a^{2}\right)^{-1 / 2}$ and $\operatorname{arc} \sinh (z / a)$ do have branch point singularities at this point. In effect, the function is $(z)$ when added to $c(z)$ eliminates the branch cut which terminates at $z=+i a$ and reinforces the branch cut which terminates at $z=-i a$. It is this elimination which allows us to use contour integration in the upper half-plane, and is vital to the whole method.

In Section 2 we discuss the general theory of constructing functions $\phi(z)=$ $c(z)+i s(z)$ satisfying (i) and (ii) with respect to a given $c(x)$.

In subsequent sections we illustrate, mainly by use of examples, how the method may be applied, with various modifications, to several simple and familiar critical functions $c(z)$.

2. Construction of Analytic Functions $\phi(z)=c(z)+i s(z)$. In this section we are concerned with the following problem. Given a real analytic function $c(x)$, find a complex analytic function $\phi(z)$ having the properties:

$$
\begin{aligned}
& \text { (i) } \operatorname{Re}(\phi(z))=c(z), z \text { real, } \\
& \text { (ii) } \phi(z) \text { has no singularities in } \operatorname{Im}(z)>0 \text {. }
\end{aligned}
$$

A basic theoretical approach to this Dirichlet problem is provided by Cauchy

\footnotetext{
*** The contour must not extend so far as to encounter nonanalytic behaviour from $w(z)$.
} 
integral and Hilbert transform theory. The following theorem summarizes some results of relevance here (Tricomi [10, Section 4.2]; Muskhelishvili [9, Section 4.2]). Following conventional practice we denote the Cauchy principal value of an integral by $P$ and the Hilbert transform operator $H_{x}\{\quad\}$ is defined by

$$
H_{x}\{f\}=\frac{1}{\pi} P \int_{-\infty}^{\infty} \frac{f(t)}{t-x} d t .
$$

Theorem 1. (i) If the real function $c(x)$ is $L_{p}(-\infty, \infty), p>1$, then

$$
s(x)=-H_{x}\{c\}
$$

defines a conjugate function for almost all $x$ which is also $L_{p}(-\infty, \infty), p>1$, and

$$
c(x)=H_{x}\{s\}
$$

for almost all $x$.

(ii) Associated with the conjugate pair $c(x), s(x)$ is the function

$$
\phi(z)=\frac{1}{2 \pi i} \int_{-\infty}^{\infty} \frac{c(t)+i s(t)}{t-z} d t .
$$

This function has the properties:

$$
\begin{gathered}
\phi(z) \text { is analytic for } \operatorname{Im}(z)>0, \\
\phi(x+i 0)=c(x)+i s(x) .
\end{gathered}
$$

Further, Muskhelishvili [9] shows under less general conditions that

$$
\phi(z)=\frac{1}{\pi i} \int_{-\infty}^{\infty} \frac{c(t)}{t-z} d t .
$$

Thus if $c(x)$ satisfies the specified integrability condition, a conjugate function $s(x)$ can be found from the Hilbert transform of $c(x)$. Associated with this conjugate pair is the desired analytic function $\phi(z)$. The problem of finding $\phi(z)$ is essentially solved if either the Hilbert transform of $c(x)$ or the Cauchy integral (2.9) can be evaluated analytically.

In the subsequent treatment we make use of two corollaries of this theorem.

Corollary 1. If $\phi(z)$ is analytic for all $\operatorname{Im}(z)>0$ and if $c(x)=\operatorname{Re}(\phi(x))$ is $L_{p}(-\infty, \infty), p>1$, then $s(x)=\operatorname{Im}(\phi(x))-\operatorname{Im}(\phi(\infty))$ and $c(x)$ are Hilbert transform pairs.

Corollary 2. If $c(x)$ and $s(x)$ are Hilbert transform pairs $L_{p}(-\infty, \infty)$ and if $c(x)$ and $s(x)$ are both analytic on the real axis, then the function $\phi(z)$ defined as the analytic continuation of $c(x)+i s(x)$ in the upper half-plane is identical with the function $\phi(z)$ defined by (2.6) or (2.9) and has no singularities in $\operatorname{Im}(z) \geqq 0$.

In applying Corollary 2, we have to be careful to note that in general the analytic continuation of either $c(x)$ or $s(x)$ separately is not defined unambiguously. Thus if we write

$$
\phi(z)=c(z)+i s(z),
$$

both $c(z)$ and $s(z)$ may have branch singularities in the upper half-plane which eliminate each other. It is important to choose $c(z)$ and $s(z)$ so that the branch cuts in the upper half-plane coincide, for Eq. (2.10) to represent $\phi(z)$. 
As an example we construct $s(x)$ and $\phi(z)$ corresponding to the function

$$
c(x)=\frac{1}{\left(x^{2}+a^{2}\right)^{1 / 2}}
$$

of the example in Section 1. Applying Eq. (2.4) of Theorem 1 we find

$$
\begin{aligned}
s(x) & =-\frac{1}{\pi} P \int_{-\infty}^{\infty} \frac{1}{\left(t^{2}+a^{2}\right)^{1 / 2}(t-x)} d t \\
& =\frac{2}{\pi\left(x^{2}+a^{2}\right)^{1 / 2}} \operatorname{arcsinh}(x / a) .
\end{aligned}
$$

In order to apply Corollary 2 , we define

$$
\begin{aligned}
& c(z)=\frac{1}{\left(z^{2}+a^{2}\right)^{1 / 2}}, \\
& s(z)=\frac{2}{\pi\left(z^{2}+a^{2}\right)^{1 / 2}} \operatorname{arcsinh}(z / a),
\end{aligned}
$$

which are analytical continuations of $c(x)$ and $s(x)$ respectively. We note that in accordance with standard definitions (Abramowitz and Stegun [2]) the branch cuts of both $c(z)$ and $s(z)$ in the upper half-plane coincide, lying immediately to the left of the line $x=0, y>a$. Thus the function $\phi(z)$ is given by

$$
\phi(z)=c(z)+i s(z)=\frac{1}{\left(z^{2}+a^{2}\right)^{1 / 2}}\left\{1+\frac{2 i}{\pi} \operatorname{arcsinh}(z / a)\right\},
$$

and according to Corollary 2 , this function has no singularities in $\operatorname{Im}(z) \geqq 0$.

Some examples of analytic Hilbert transform pairs satisfying the $L_{p}$ integrability criterion are given in the upper section of Table 1. When the corresponding $\Phi(z)$ is easily recognized to be reducible to a simpler form than $c(z)+i s(z)$, this function of $z$ is also entered. Table 2 lists some general properties of Hilbert transforms, useful for extending known transforms. Of special interest is the reciprocity, which enables columns in transform pair tables to be interchanged with a relative change in sign. Differentiation also generates a new transform pair. In some cases new transform pairs can be obtained by integration; for example the sixth entry in Table 1 can be obtained by integrating the first entry and inserting a factor $x^{-1}$ (or $z^{-1}$ in $\phi(z))$ for convergence.

Tables of Hilbert transforms useful for the present application are limited (Erdélyi et al. [5, Vol. 2], Macdonald and Brachman [7]), since only transforms of critical factors are of real interest here. If the appropriate indefinite integral is found listed in conventional integral tables, it may be used to deduce the Hilbert transform. Another method is to recognize that Fourier sine and cosine transforms of the same function are formally Hilbert transform pairs. For example, the first four entries in Table 1 may be obtained in this way from standard tables of sine and cosine transforms (see Erdélyi et al. [5, Vol. I]).

Rational functions play a special role in the subsequent theory. Although the Hilbert transform of a rational function is not essential for the applications described here, we do require the Hilbert transform of the product of a rational func- 
tion $r(x)$ with a function $c(x)$ whose Hilbert transform is known.

We consider rational functions $r(z)$ which have no poles on the real axis, and which are real for real $z$. To simplify the discussion, it is convenient to restrict attention to rational functions in which the degree of the denominator exceeds that of the numerator. This restriction is not always necessary but avoids complications of a nonessential character. If $r(z)$ has poles of order $n_{s}$ at $z_{s}$ and $\bar{z}_{s},(s=1, \cdots, n$, $\left.\operatorname{Im}\left(z_{s}\right)>0\right), r(z)$ may be expanded in partial fractions as follows:

$$
\begin{aligned}
r(z)=\sum_{s=1}^{n}\left\{\frac{a_{-n}^{(s)}}{\left(z-z_{s}\right)^{n_{s}}}+\frac{a_{-n_{s}+1}^{(s)}}{\left(z-z_{s}\right)^{n_{s}-1}}+\cdots+\frac{a_{-1}^{(s)}}{z-z_{s}}\right. \\
\left.\quad+\frac{\bar{a}_{-n_{s}}^{(s)}}{\left(z-\bar{z}_{s}\right)^{n_{s}}}+\frac{\bar{a}_{-n_{s}+1}^{(s)}}{\left(z-\bar{z}_{s}\right)^{n_{s}-1}}+\cdots+\frac{\bar{a}_{-1}^{(s)}}{z-\bar{z}_{s}}\right\}
\end{aligned}
$$

where bars denote complex conjugates. We define

$$
\psi_{s}(z)=\left\{\frac{a_{-n_{s}}^{(s)}}{\left(z-z_{s}\right)^{n_{s}}}+\cdots+\frac{a_{-1}^{(s)}}{z-z_{s}}\right\}-\left\{\frac{\bar{a}_{-n_{s}}^{(s)}}{\left(z-\bar{z}_{s}\right)^{n_{s}}}+\cdots+\frac{\bar{a}_{-1}^{(s)}}{z-\bar{z}_{s}}\right\}
$$

and

$$
\begin{aligned}
\tilde{\phi}(z) & =r(z)-\sum_{s=1}^{n} \psi_{s}(z) \\
& =2 \sum_{s=1}^{n}\left[\frac{\bar{a}_{-n_{s}}^{(s)}}{\left(z-\bar{z}_{s}\right)^{n_{s}}}+\frac{\bar{a}_{-n_{s}+1}^{(s)}}{\left(z-\bar{z}_{s}\right)^{n_{s}-1}}+\cdots+\frac{\bar{a}_{-1}^{(s)}}{z-\bar{z}_{s}}\right] .
\end{aligned}
$$

Clearly $\operatorname{Re}\left(\psi_{s}(x)\right)=0$ and $\operatorname{Re}(\tilde{\phi}(x))=r(x)$. Also $\tilde{\phi}(z)$ is analytic for $\operatorname{Im}(z) \geqq 0$ having poles only at the points $z=\bar{z}_{s}$ in the lower half-plane. It follows from Corollary 1 that the Hilbert transform of $r(x)$ is $\operatorname{Im} \tilde{\phi}(x)$. Simple meromorphic functions can sometimes be handled in the same way by using the Mittag-Leffler expansion instead of the partial fraction expansion (see entries 11 and 12 in Table 1).

We now turn to the problem of determining the Hilbert transform of

$$
c(x)=r(x) c^{*}(x)
$$

where the Hilbert transform $s^{*}(x)$ of $-c^{*}(x)$ and the corresponding function

$$
\phi^{*}(z)=c^{*}(z)+i s^{*}(z)
$$

are known, and $r(x)$ is given by (2.16) above. We apply the same principles as in the case of the rational function, with the modification that Laurent expansions of $r(z) \phi^{*}(z)$ are used instead of the partial fraction expansion of $r(z)$. This expansion, about one of the poles $z_{s}$ of $r(z)$, is

$$
\begin{aligned}
\psi(z)=\phi^{*}(z) r(z)=\frac{b_{-n_{s}}^{(s)}}{\left(z-z_{s}\right)^{n_{s}}} & +\frac{b_{-n s+1}^{(s)}}{\left(z-z_{s}\right)^{n_{s}-1}}+\cdots+\frac{b_{-1}^{(s)}}{z-z_{s}} \\
& +b_{0}^{\left({ }^{(s)}\right.}+b_{1}^{(s)}\left(z-z_{s}\right)+\cdots
\end{aligned}
$$

where the numbers $b_{i}{ }^{(s)}\left(i=-n_{s},-n_{s}+1, \cdots,-1\right)$ are calculated from the first $n_{s}$ derivatives of $\phi^{*}(z)$ at $z_{s}$ and the coefficients $a_{i}{ }^{\left({ }{ }_{s}\right)}\left(i=-n_{s},-n_{s}+1, \cdots,-1\right)$. In analogy to (2.17), we define 


$$
\begin{aligned}
\psi_{s}(z)= & \frac{b_{-n_{s}}^{(s)}}{\left(z-z_{s}\right)^{n_{s}}}+\frac{b_{-n_{s}+1}^{(s)}}{\left(z-z_{s}\right)^{n_{s-1}}}+\cdots+\frac{b_{-1}^{(s)}}{z-z_{s}} \\
& -\left\{\frac{\bar{b}_{-n_{s}}^{(s)}}{\left(z-\bar{z}_{s}\right)^{n_{s}}}+\frac{\bar{b}_{-n_{s}+1}^{(s)}}{\left(z-\bar{z}_{s}\right)^{n_{s-1}}}+\cdots+\frac{\bar{b}_{-1}^{(s)}}{z-\bar{z}_{s}}\right\} .
\end{aligned}
$$

$\psi_{s}(z)$ has a pole at $z=z_{s}$ of precisely the same nature as the corresponding pole of $\psi(z)$, and $\operatorname{Re}\left(\psi_{s}(z)\right)=0$ when $z$ is real.

We now define

$$
\phi(z)=\psi(z)-\sum_{s=1}^{n} \psi_{s}(z)=\phi^{*}(z) r(z)-\sum_{s=1}^{n} \psi_{s}(z) .
$$

$\phi^{*}(z)$ by definition is analytic in the upper half-plane. Thus the only possible singularities of $\psi(z)$ in the upper half-plane are poles at $z=z_{s}(s=1, \cdots, n)$. Each of these is precisely cancelled by the subtraction of the corresponding $\psi_{s}(z)$. Thus $\phi(z)$ is analytic for $\operatorname{Im}(z)>0$. that

Moreover, when $z$ is real, $r(z)$ is real, $\operatorname{Re}\left(\phi^{*}(z)\right)=c^{*}(z)$ and $\operatorname{Re}\left(\psi_{s}(z)\right)=0$, so

$$
\operatorname{Re}(\phi(x))=c^{*}(x) r(x) .
$$

Thus $\phi(z)$ satisfies the conditions of Corollary 1 and the Hilbert transform of $r(x) c^{*}(x)$ is $\operatorname{Im} \phi(x)$ where $\phi(x)$ is given by (2.23).

As an example we compute the Hilbert transform of $c^{*}(x) /(x-\beta)(x-\bar{\beta})$, $\operatorname{Im}(\beta)>0$ where the Hilbert transform of $c^{*}(x)$ is $-s^{*}(x)$ and

$$
\phi^{*}(z)=c^{*}(z)+i s^{*}(z)
$$

is analytic for $\operatorname{Im}(z) \geqq 0$. The Laurent expansion of $\psi(z)=\phi^{*}(z) r(z)$ about $z=\beta$ is

$$
\psi(z)=\frac{\phi^{*}(\beta)}{(\beta-\bar{\beta})} \frac{1}{(z-\beta)}+b_{0}+b_{1}(z-\beta)+\cdots .
$$

Thus

$$
\psi_{s}(z)=\frac{\phi^{*}(\beta)}{(\beta-\bar{\beta})(z-\beta)}+\frac{\overline{\phi^{*}(\beta)}}{(\beta-\bar{\beta})(z-\bar{\beta})}
$$

and

$$
\phi(z)=\phi^{*}(z) r(z)-\frac{\phi^{*}(\beta)}{(\beta-\bar{\beta})(z-\beta)}-\frac{\overline{\phi^{*}(\beta)}}{(\beta-\bar{\beta})(z-\bar{\beta})}
$$

which may be put in the form

$$
\phi(z)=\frac{1}{\beta-\bar{\beta}}\left\{\frac{\phi^{*}(z)-\phi^{*}(\beta)}{z-\beta}-\frac{\phi^{*}(z)+\overline{\phi^{*}(\beta)}}{z-\bar{\beta}}\right\} .
$$

This function is analytic for $\operatorname{Im}(z) \geqq 0$ and the Hilbert transform of $c^{*}(x)$ $\cdot((x-\beta)(x-\bar{\beta}))^{-1}$ is $-\operatorname{Im}(\phi(x))$.

Up to this point we have considered the Hilbert transform and associated function $\phi(z)$ only of functions $c(x)$ which are analytic for all real $x$, and $L_{p}(-\infty, \infty)$, $p>1$. The integrability condition ensures that the Hilbert transform exists. 
For functions $c(x)$ which are finite over a segment of the real axis, the integrability condition can be met by defining a function equal to $c(x)$ in some interval $(a, b)$ and zero elsewhere. The Hilbert transform then exists and Erdélyi et al. [5, Vol. 2] tabulates transforms of this type. However, because of the discontinuities at the end points $a, b$ of the interval, the corresponding $\phi(z)$ exhibits nonanalytic behavior at these points. Transforms of this type have not been employed in the applications reported here, but they may be used provided the end points of the interval of integration are not close to the singularities at $x=a, b$.

While the $L_{p}$ integrability condition, by ensuring the existence of a Hilbert transform, guarantees that an associated $\phi(z)$ exists, this condition is by no means necessary. For example, addition of constants to $c(x)$ and $s(x)$ destroys the $L_{p}$ integrability, but alters the associated $\phi(z)$ only by the trivial addition of a complex constant. A list of some functions $c(x), s(x)$ which are not $L_{p}(-\infty, \infty), p>1$, but are Hilbert transform pairs, appears in the lower part of Table 1 . In each case the associated function $\phi(z)$ is given which satisfies,

$$
\begin{gathered}
\operatorname{Re}(\phi(x))=c(x), \\
\operatorname{Im}(\phi(x))=s(x), \\
\phi(z) \text { is analytic for } \operatorname{Im} z \geqq 0 .
\end{gathered}
$$

In some cases such as $c(x)=\cos x$, there is an essential singularity at infinity in both $c(z)$ and in $s(z)=\sin x$. Nevertheless, the associated function $\phi(z)=e^{i z}$ is regular in $\operatorname{Im}(z) \geqq 0$. In the applications described in Section 5 , this is important. However in other applications, the property of interest is that singularities in $\phi(z)$ in a particular finite region of the upper half-plane should not occur, and it is immaterial whether or not $\phi(z)$ has a singularity at infinity. For example, from Tables 1 and 2 we find that corresponding to

$$
c(x)=(1 / 2 x) \ln \left(1+x^{2} / a^{2}\right)
$$

is the conjugate function,

$$
s(x)=-(1 / x) \arctan (x / a), \quad a>0,
$$

and

$$
\phi(z)=(1 / z) \ln (1-i z / a)
$$

is analytic in $\operatorname{Im}(z) \geqq 0$ with

$$
\operatorname{Re}(\phi(x))=c(x) ; \quad \operatorname{Im}(\phi(x))=s(x) .
$$

If we are interested in a function having a critical factor $\ln \left(1+x^{2} / a^{2}\right)$ we may well dispense with the $1 / x$ factor. In this case it follows that if we define

$$
\begin{aligned}
& \tilde{c}(x)=(1 / 2) \ln \left(1+x^{2} / a^{2}\right), \\
& \tilde{s}(x)=-\arctan x / a,
\end{aligned}
$$

and

$$
\varphi(z)=\ln (1-i z / a)
$$

we have as before that 


$$
\operatorname{Re}(\tilde{\phi}(x))=\tilde{c}(x) ; \quad \operatorname{Im}(\tilde{\phi}(z))=\tilde{s}(x)
$$

and now $\tilde{\phi}(z)$ has no singularities in any finite region of the upper half plane (but does have a singularity at infinity). The functions $\tilde{c}(x)$ and $\tilde{s}(x)$ are not Hilbert transform pairs (except perhaps in some generalised sense). However in the applications given here the pair $\tilde{c}(x), \tilde{\phi}(z)$ is just as useful as a basis for the calculation as the pair $c(x), \phi(x)$.

Consequently, although Hilbert transform theory forms a convenient basis for the procedures described here, some of the restrictions associated with this theory are not relevant to the applications.

3. Application to Numerical Quadrature (General Remarks). In the previous section we showed that for a very wide class of analytic functions $c(x)$, it is possible to define corresponding functions $s(x)$ and $\phi(z)$ having the properties

(i) $\phi(z)$ has no singularities in the upper half-plane,

(ii) $\operatorname{Re} \phi(z)=c(z), z$ real.

Consequently if we define a contour $C$ which connects $z=-1$ to $z=1$ in the upper half-plane and define the region $R$ to be that bounded by $C$ and the real axis, we may write

$$
\int_{-1}^{1} c(x) d x=\operatorname{Re} \int_{-1}^{1} \phi(x) d x=\operatorname{Re} \int_{c} \phi(z) d z .
$$

Moreover, if $w(x)$ is a real analytic function and $w(z)$ has either no singularities or only poles in the region $R$, we may write

$$
\begin{aligned}
\int_{-1}^{1} w(x) c(x) d x & =\operatorname{Re} \int_{-1}^{1} w(x) \phi(x) d x \\
& =\operatorname{Re} \int_{c} w(z) \phi(z) d z+2 \pi i \sum_{R} R(w \phi)
\end{aligned}
$$

where $\sum_{R} R(w \phi)$ stands for the sum of the residues of the poles of $w(z) \phi(z)$ in the region $R$. In principle therefore, when

$$
f(x)=w(x) c(x)
$$

we may generally apply transformation (3.2) and evaluate, numerically or otherwise, the expression on the right-hand side instead of the expression on the left-hand side. In many cases illustrated by examples in the following sections, the latter calculation is significantly easier than the former.

We must emphasise that in practice this choice exists only if the integration required to calculate $\phi(z)$ from $c(x)$ has been carried out analytically and some simple analytic form for $\phi(z)$ is known. Thus while the theory as presented in the previous section is quite general, this particular application of this theory is restricted in practice.

There are two distinct types of limitations to the use of this theory. The first is that it should be possible to use it and the second that it should be desirable to use it. These may be summarised in a nonrigorous manner as follows:

(i) Practical Possibility. It must be possible to factorise $f(x), f(x)=w(x) c(x)$, in such a manner that: $c(x)$ is a function for which a simple analytic expression for 
the corresponding $\phi(z)$ is known; and $w(z)$ has no singularities other than poles in $R$.

(ii) Desirability. The function $f(x)$ should be "difficult" to integrate along the real axis (possibly because $c(z)$ has branch singularities close to the real axis), but the function $w(z) \phi(z)$ should be "easy" to integrate along the contour $C$ and any residues at poles of $w(z) \phi(z)$ within $R$ should be easy to calculate analytically.

The above remarks are clearly only a qualitative guide to the limitations. Obviously the terms "difficult," "easy," and "simple" have to be taken in a relative sense. If $c(x)$ is given, the function $\phi(z)$ may be expressed

$$
\phi(z)=\frac{1}{\pi i} \int_{-\infty}^{\infty} \frac{c(t)}{t-z} d z
$$

and in other contexts this might be described as "a simple analytical expression." In this context, where the original problem consists of an integration which may be simpler than this, this form is not simple enough.

So far as "difficult" and "easy" to integrate are concerned, this depends on the locations of and the nature of the singularities of $f(z)$. In general a singularity close to the integration path is associated with a peak in the integrand on the path close to the singularities. This has the effect that standard quadrature routines require a large number of function evaluations to attain an approximation of particular accuracy. There is also the subsidiary effect of the accuracy being limited by roundoff error in function evaluations near the peak. This is not the whole story. The function $\cos (n x)$ has no singularities, except for an essential singularity at infinity. This single singularity even though it is at infinity causes "difficult" integration if $n$ is large. However, the relative difficulty depends also on the facilities available to the user. If a special quadrature routine is available designed to take into account as a weighting function the critical factor, then instead of being "difficult," a quadrature may be "easy."

4. Examples Using a Semicircular Contour. In this section we apply the method outlined in Section 3 to specific cases in which there is a branch singularity in the integrand close to the interval of integration. In this section we use the same semicircular contour $C$ to evaluate the contour integrals. Since a general discussion is rather difficult to follow we present the material in the form of examples together with a discussion of that particular example.

Example 1.

$$
I_{1}=\int_{-1}^{1} \frac{g(x)}{\left(a^{2}+x^{2}\right)^{1 / 2}\left((x-\lambda)^{2}+\mu^{2}\right)} d x, \quad \mu>0,
$$

where $g(x)$ is an analytic function having the same characteristics as $w(x)$, that is it is not a numerically critical factor. This integrand may be written

$$
f(z)=g(z) /\left(\left(z^{2}+a^{2}\right)^{1 / 2}(z-\beta)(z-\bar{\beta})\right)
$$

and has branch singularities at $z= \pm i a$ and simple poles at $z=\beta=\lambda+i \mu$ and at $z=\bar{\beta}=\lambda-i_{\mu}$. Unless one of these singularities is close to the interval $[-1,1]$, the numerical quadrature is straightforward and should be carried out directly. If this is not the case, and if $a$ is small there is at the outset a choice in what we consider to be the critical factor. On one hand we may choose 


$$
c^{*}(x)=1 /\left(x^{2}+a^{2}\right)^{1 / 2} ; \quad w^{*}(x)=g(x) /(x-\beta)(x-\bar{\beta})
$$

and proceed along the lines described in the previous section. Reference to Table 1 indicates that

$$
\phi^{*}(z)=\frac{1}{\left(z^{2}+a^{2}\right)^{1 / 2}}\left\{1+\frac{2 i}{\pi} \operatorname{arcsinh}(z / a)\right\} .
$$

The residue of the function $\phi^{*}(z) w^{*}(z)$ at the pole $z=\beta$ is $\phi^{*}(\beta) g(\beta) / 2 i_{\mu}$. Thus we find

$$
I_{1}=\operatorname{Re} \int_{c} \frac{g(z) \phi^{*}(z)}{(z-\beta)(z-\bar{\beta})} d z+\operatorname{Re}(2 \pi i R)
$$

where

$$
\begin{aligned}
& R=\phi^{*}(\beta) g(\beta) / 2 i \mu, \quad|\beta|<1, \\
& R=0, \quad|\beta|>1 .
\end{aligned}
$$

This is the first choice. The second is to include the rational function $\left\{(x-\lambda)^{2}+\mu^{2}\right\}^{-1}$ as part of the critical factor. Thus

$$
c(x)=1 /\left(x^{2}+a^{2}\right)^{1 / 2}(x-\beta)(x-\bar{\beta}) ; \quad w(x)=g(x) .
$$

Since the Hilbert transform of $c^{*}(x)$ is known together with the corresponding function $\phi^{*}(z)$, the function $\phi(z)$ which corresponds to $c(x)$ may be determined using the theory provided in the second half of Section 2. This particular calculation is carried out there as an example. The result (Eq. (2.26)) is

$$
\phi(z)=\frac{1}{2 i \mu}\left\{\frac{\phi^{*}(z)-\phi^{*}(\beta)}{z-\beta}-\frac{\phi^{*}(z)+\overline{\phi^{*}(\beta)}}{z-\bar{\beta}}\right\} .
$$

The function $\phi(z) g(z)$ has no singularities in the region $R$. Consequently,

$$
\begin{aligned}
I_{1} & =\operatorname{Re} \int_{c} w^{*}(z) \phi^{*}(z) d z=\int_{c} w(z) \phi(z) d z \\
& =\operatorname{Re} \int_{c} \frac{g(z)}{2 i \mu}\left[\frac{\phi^{*}(\beta)-\phi^{*}(z)}{z-\beta}-\frac{\phi^{*}(z)+\overline{\phi^{*}(\beta)}}{z-\bar{\beta}}\right] d z .
\end{aligned}
$$

The foregoing results (4.5) and (4.9) are analytic in nature and are valid whatever the values of $a$ and $\beta$ so long as $a>0, \mu>0$. If $\beta$ lies on the semicircle $C$, i.e. $|\beta|=1$, both (4.5) and (4.9) have to be interpreted in a standard limiting sense. Moreover, (4.9) is a simple analytic consequence of (4.5) and may be obtained by expressing the residue $R$ as a contour integral using as closed contour the semicircle $C$ and line interval $[-1,1]$.

In several numerical cases, taking $g(z)=1.0, a$ small, and various locations for the pole, the slightly more complicated form (4.9) is easier to integrate numerically than (4.5). A typical example is one with $a=0.001$ and $\beta=0.4+0.1 i$. Here to obtain an accuracy $\epsilon=10^{-5}$ the integration in (4.1) required 1927 (ASM) or $16385(\mathrm{R})$ function evaluations of a real function. That in (4.5) required 247(ASM) or 129(R) complex function evaluations and that in (4.9) required 163(ASM) or $65(\mathrm{R})$ complex function evaluations. This is despite the fact that the same absolute accuracy $10^{-5} \mathrm{in}$ 
the final result was required and the integral in (4.5) whose value is -1.28 is much smaller than the integral, $I_{1}=140.11$, in (4.9). The effect of removing all the poles from the upper half-plane, rather than relying on the residue term is such that in this case a relative accuracy of $10^{-7}$ is obtained with less work than a relative accuracy of $10^{-5}$.

The case in which $|\beta|$ is close to 1 , so that the pole is close to the contour $C$, need not arise in practice, as under these circumstances a different contour $C^{\prime}$ would be chosen. However, if for some other reason it is convenient to use the contour $C$ unduly large round-off errors in function evaluation may occur at points close to the point $\beta$. The authors have found in practice that accurate results may still be obtained using (4.9), but not (4.5). The situation is analogous to integrating the function $(\sin z) / z$ and the function $(\cos z) / z$ respectively along a contour close to or even through the origin. In one case, corresponding to (4.9) the magnitude of the function is not large at the points where the function value is subject to inaccuracy and these errors are averaged out in the integration. In the other case corresponding to (4.5) the result depends on adding large numbers having opposite signs where these numbers are inaccurate, and this leads to completely unreliable results.

The conclusion in this example is that under very wide conditions the use of (4.9) is to be preferred to the use of (4.5), the additional analytic work required in calculating the Hilbert transform of the product $c^{*}(x) r(x)$ rather than that of $c^{*}(x)$ being rewarded both by an easier numerical integration and a mitigation of possible effect of round-off error.

Example 2.

$$
I_{2}=\int_{-1}^{1} g(x) \frac{\ln \left(1+(x / a)^{2}\right)}{x^{2}+a^{2}} d x
$$

where $a$ is small and $g(x)$ is as before not numerically critical. Here we may proceed in precisely the same manner as in Example 1. There are branch singularities at $z= \pm i a$ being the coalescence of a logarithmic branch point and a simple pole. Reference to Tables 1 and 2 indicates that corresponding to the function

$$
\tilde{c}(x)=(1 / 2 x) \ln \left(1+x^{2} / a^{2}\right)
$$

is the function

$$
\tilde{\phi}(z)=(1 / z) \ln (1-i z / a)
$$

which has no singularities in $\operatorname{Im}(z) \geqq 0$. The discussion at the end of Section 2 shows that if we are prepared to allow a function having no singularities in any finite region of the upper half-plane (but one at infinity) we may set

$$
c(x)=\ln \left(1+x^{2} / a^{2}\right)
$$

and

$$
\phi(z)=2 \ln (1-i z / a) .
$$

The residue of $\phi(z) g(z) /\left(z^{2}+a^{2}\right)$ at $z=+i a$ is

$$
R=(g(i a) \ln 2) / i a \text {. }
$$




$$
I_{2}=\operatorname{Re}\left[\int_{c} \frac{g(z) \phi(z)}{z^{2}+a^{2}} d z+\frac{2 \pi}{a} g(i a) \ln 2\right], \quad a<1 .
$$

A calculation regarding

$$
\left[\frac{x}{x^{2}+a^{2}}\right]\left[\frac{1}{x} \ln \left(1+\frac{x^{2}}{a^{2}}\right)\right]
$$

as the critical function leads to the result

$$
I_{2}=\operatorname{Re}\left[\int_{c} 2 g(z)\left\{\frac{\ln (1-i z / a)+(i z / a) \ln 2}{z^{2}+a^{2}}\right\} d z\right], \quad a<1,
$$

which is analogous to Eq. (4.9). Numerical examples give results following much the same pattern as in Example 1. For example, with $g(x)=1.0$ and $a=10^{-2}$, an absolute accuracy of $10^{-4}$ was obtained in both (4.16) and (4.17) using 55(ASM) or $17(\mathrm{R})$ complex function evaluations. The real integral (4.10) required 1135(ASM) or $2049(\mathrm{R})$ real function evaluations.

The examples chosen have contained a simple critical factor whose Hilbert transform was known, and the whole integral was converted into a single complex integral around a simple contour. If the singularities of the integrand of the real integral are distributed in the direction of the real axis the real integral can be split up and each part treated separately. In this way knowledge of the Hilbert transform of simple functions, rather than their products, may suffice. For example, suppose

$$
I=\int_{-1}^{1} g(x) \frac{\ln \left(1+\left(x-\frac{1}{2}\right)^{2} / a^{2}\right)}{\left(b^{2}+\left(x+\frac{1}{2}\right)^{2}\right)^{1 / 2}} d x
$$

is required, where $a$ and $b$ are both small. The authors do not know the Hilbert transform of

$$
\frac{\ln \left(1+\left(x-\frac{1}{2}\right)^{2} / a^{2}\right)}{\left(b^{2}+\left(x+\frac{1}{2}\right)^{2}\right)^{1 / 2}}
$$

so they cannot use the method directly. However, writing

$$
I=J_{1}+J_{2}
$$

with

$$
\begin{aligned}
& J_{1}=\int_{-1}^{0}\left\{g(x) \ln \left[1+\left(x-\frac{1}{2}\right)^{2} / a^{2}\right]\right\} \frac{1}{\left(b^{2}+\left(x+\frac{1}{2}\right)^{2}\right)^{1 / 2}} d x, \\
& J_{2}=\int_{0}^{1}\left\{\frac{g(x)}{\left(b^{2}+\left(x+\frac{1}{2}\right)^{2}\right)^{1 / 2}}\right\} \ln \left[1+\left(x-\frac{1}{2}\right)^{2} / a^{2}\right] d x
\end{aligned}
$$

separates the singularities, and the method can be applied to each integral in turn. The appropriate $w(x)$ function to use in each case is contained in the curly brackets in (4.20), (4.21).

The choice of a contour, together with the appropriate quadrature rule is not as unimportant as it might seem at first. The points for function evaluation have to be calculated and a contour and rule should be chosen with this in mind. A set of straight line segments, together with any standard quadrature rule for each, present 
no difficulty. In this section we have used a semicircular contour. The points for function evaluation are of the form $\exp \left[\pi i t_{j}\right], 0 \leqq t_{j} \leqq 1$. If we were to use a rule involving a high order Gaussian quadrature, a large number of calls to the exponential routine would be necessary. On the other hand, a rule depending on equally spaced points $t_{j}=j / n$ requires only one call to the exponential routine to calculate $e^{\pi i / n}$. The other points are simply powers of this $\exp \left[\pi i t_{j}\right]=\left(\exp \left[\pi i t_{1}\right]\right)^{j}$. In practice Romberg integration was considered advantageous.

It is quite pertinent to suggest that some other contour such as three sides of a square having $[-1,1]$ as base is more appropriate, since there is a wider scope for choice of quadrature rules. Any such choice depends on the location of the singularities. In general a circular segment having the most influential singularity as center has the property that the integrand varies smoothly along the contour. In the examples in this section $\phi(z)$ has a branch singularity at $z=-i a$, which is close to the origin. Thus a semicircle centered at the origin is appropriate to these problems.

5. Examples Using an Infinite Contour. In this section we consider examples in which the function exhibits oscillating behavior of a simple nature on the interval of integration. This may be due to an essential singularity at infinity or due to an infinite sequence of isolated singularities. We give an example of each.

Example 3. We consider first the Fourier coefficient integral

$$
\int_{-1}^{1} w(x) \cos (n x) d x
$$

where $n$ is taken to be large, e.g. $n=10$, and need not be a multiple of $\pi$.

We refer to Table 1 and find

$$
\begin{aligned}
& c(x)=\cos (n x), \\
& \phi(z)=e^{i n z} .
\end{aligned}
$$

Thus, so long as the function $w(z)$ has no singularities other than poles in the region $R$ (bounded by $C$ and the interval $[-1,1]$ ) we have as before

$$
\int_{-1}^{1} w(x) \cos n x d x=\operatorname{Re}\left\{\int_{c} w(z) e^{i n z} d z+2 \pi i \sum_{R} R(w \phi)\right\} .
$$

In this problem, attention has to be focused on the choice of contour $C$. In any line segment (not parallel to the $y$ axis) the function $e^{\text {inz }}$ has oscillating behavior. However, along a line segment parallel to the $y$ axis, the function $e^{i n z}$ is decaying exponentially and has no oscillatory behavior. Moreover, if $\operatorname{Im}(z)$ is large and positive, the function $e^{i n z}$ is small in magnitude. Thus a suitable contour $C$ might be three sides of the rectangle of height $L$ having the interval $[-1,1]$ as base, $L$ being chosen to be large enough so that the contribution to the integral along the upper edge of the rectangle is smaller than the required tolerance.

To be specific we consider an example in which a pole of order two a small distance $\mu$ from the real axis presents an added difficulty to the evaluation of (5.1) numerically. We set

$$
w(x)=1 /(x-\beta)^{2}(x-\bar{\beta})^{2} \text { where } \beta=\lambda+i \mu,|\lambda|<1, \mu>0 .
$$




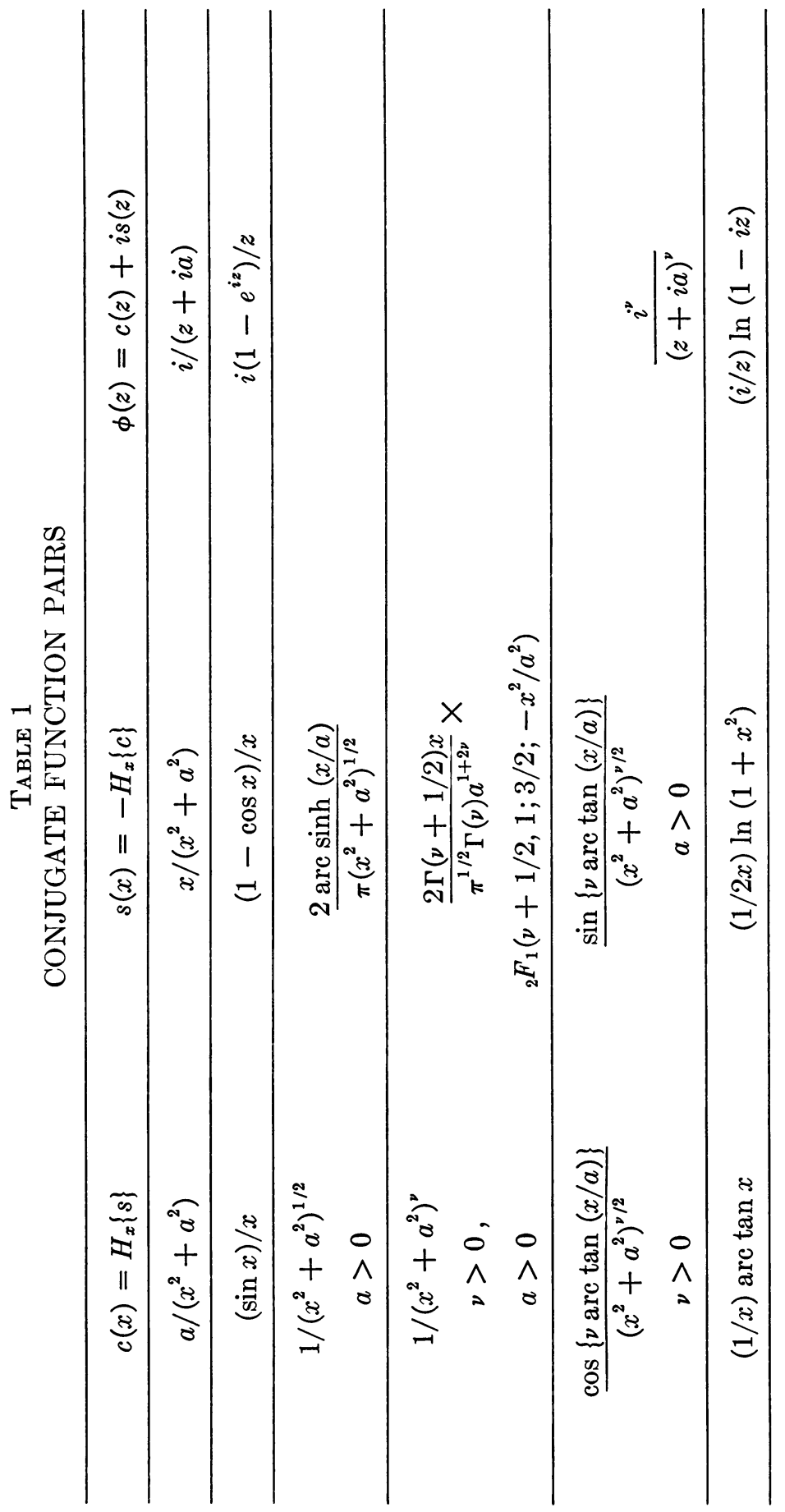




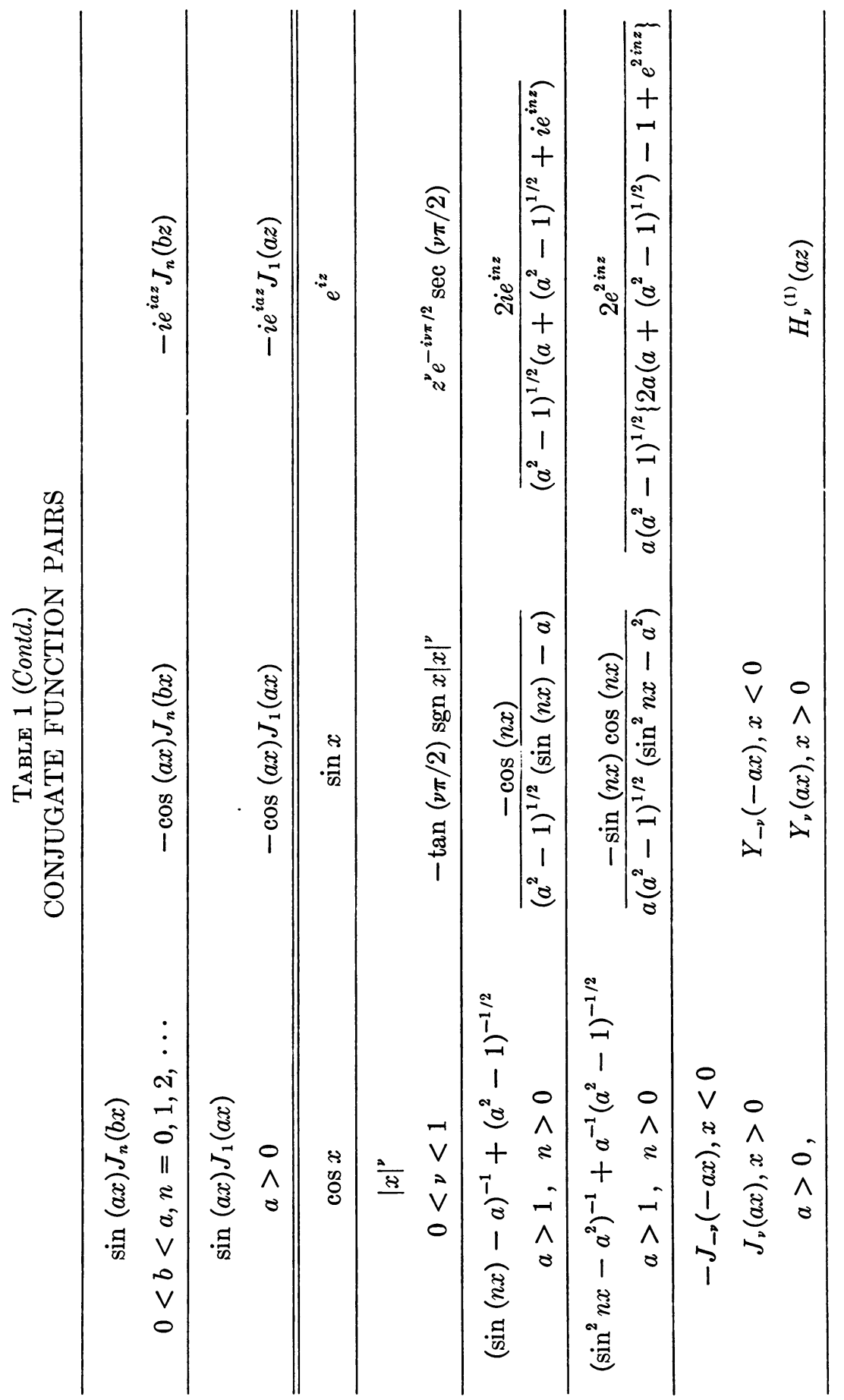


The residue of the pole of $e^{i n z} w(z)$ at $z=\beta$ is

$$
R=-\left(i / 4 \mu^{3}\right) e^{i n \beta}(1+n \mu) .
$$

This leads to the result

$$
\begin{aligned}
& \int_{-1}^{1} \frac{\cos n x}{(x-\beta)^{2}(x-\bar{\beta})^{2}} d x \\
& =\operatorname{Re}\left\{i \int_{0}^{L} e^{-n y}\left[e^{-i n} w(-1+i y)-e^{i n} w(1+i y)\right] d y\right. \\
& \left.\quad+e^{-n L} \int_{-1}^{1} e^{i n x} w(x+i L) d x+\frac{\pi}{2 \mu^{3}} e^{i n \beta}(1+n \mu)\right\} ; L>\mu .
\end{aligned}
$$

This result is of course exact. The only question is that of making a choice of $L$ and deciding whether the integrals on the right are easier to evaluate numerically than the one on the left. As mentioned above, $L$ may be chosen to make the contribution of the second integral negligible; to do this we require a value of $L$ for which

$$
2 e^{-n L} \max _{-1<x<1} w(x+i L)<\epsilon
$$

and there is no difficulty here.

TABLE 2

GENERAL PROPERTIES OF CONJUGATE FUNCTION PAIRS

\begin{tabular}{ccc}
\hline $\begin{array}{c}C(x) \\
=H_{x}\{S\}\end{array}$ & $\begin{array}{c}S(x) \\
-\end{array}$ & $\begin{array}{c}\text { Corresponding Analytic } \\
\text { Function in } \operatorname{Im}(z)>0\end{array}$ \\
\hline$C(x)$ & $S(x)$ & $\phi(z)=C(z)+i S(z)$ \\
\hline$S(x)$ & $-C(x)$ & $-i \phi(z)$ \\
\hline $\begin{array}{c}C(x+a) \\
a \text { real }\end{array}$ & $S(x+a)$ & $\phi(z+a)$ \\
\hline$C(a x)$ & $S(a x), a>0$ & $\phi(a z)$ \\
$a$ real & $-S(a x), a<0$ & $C(a z)-i S(a z)$ \\
\hline$C^{\prime}(x)$ & $S^{\prime}(x)$ & $\phi^{\prime}(z)$ \\
\hline$x C(x)$ & $x S(x)-\pi^{-1} \int_{-\infty}^{\infty} C(x) d x$ & $z \phi(z)-i \pi^{-1} \int_{-\infty}^{\infty} C(x) d x$ \\
\hline
\end{tabular}

The only other difficulty appears when we consider the first integral on the right of (5.6). The function of $y$ in square brackets is a relatively smoothly varying function. However, the factor $e^{-n y}$ is the rapidly decaying exponential function. Thus standard methods are not readily available (but could be constructed) for finite intervals. However, in this example it is more convenient to set $L=\infty$. Then 
we clearly satisfy (5.7) and we may use methods based on the Gauss-Laguerre quadrature formulas for the only remaining interval. It has been shown that a sequence of Gauss-Laguerre formulas does converge under these circumstances (see Uspensky [11]).

In a numerical example, with $\beta=0.5+0.1 i$ and $n=10 \pi$ the integral in (5.6) was evaluated using the two-point Gauss-Laguerre formula to give an overall relative accuracy of $10^{-5}$. The four-point formula gave $10^{-7}$.

There are two points which should be noticed. First the value of

$$
\int_{-1}^{1} \frac{\sin n x}{(x-\beta)^{2}(x-\bar{\beta})^{2}} d x
$$

is simply the imaginary part of the quantity in curled brackets on the right-hand side of Eq. (5.6) and can be calculated at the same time. However, if a set of Fourier coefficients are required $\int_{-1}^{1} \sin 2 \pi m x f(x) d x, m=1,2,3, \cdots$, each has to be calculated separately if this method is used. There are other methods available which would use the same function values to calculate all the members of the set, and these might be more appropriate. The second point is that, if an infinite contour is chosen, the general restriction that $w(x)$ should have no singularities in the region $R$ is of course valid, but has to be properly interpreted so far as the situation at infinity is concerned. For example $w(x)=e^{m x}$ has an essential singularity at infinity. Thus the integral

$$
\int_{-1}^{1} g(x) e^{m x} \cos n x d x=\operatorname{Re} \int_{-1}^{1} g(z) e^{(m+i n) z} d z
$$

cannot be treated in this way if $|m| \geqq|n|$, and the method may be inefficient if $|m|<|n|$. In this case, an appropriate procedure is to choose contours along which $e^{(m+i n) z}$ is exponentially decaying and has no oscillatory part. These are the lines $m y+n x= \pm n$.

Example 4 . The second example we consider here is

$$
\int_{-1}^{1} \frac{w(x)}{\sin n x-a} d x, \quad a>1,
$$

where to avoid complicating matters we suppose that $w(z)$ is regular within $|\operatorname{Re}(z)| \leqq 1, \operatorname{Im}(z) \geqq 0$. If $a$ is close to 1 and $n$ is large, the factor

$$
\tilde{c}(x)=1 /(\sin n x-a)
$$

is a critical factor, having period $2 \pi / n$ and varying in value between $-1 /(a-1)$ and $-1 /(a+1)$. A simple calculation shows that this oscillatory behavior is due to a sequence of simple poles of $\tilde{c}(z)$ situated at intervals of $2 \pi / n$ on the two lines $|\operatorname{Im}(z)|= \pm(1 / n) \cosh ^{-1}(a)$.

Before referring to the table of Hilbert transforms, we note that the function

$$
\left(1-\frac{i \cos n z}{\left(a^{2}-1\right)^{1 / 2}}\right)
$$

has zeros which coincide with the poles of $\tilde{c}(z)$ in the upper half plane but not in the lower half-plane. Thus the function 


$$
\tilde{\phi}(z)=\frac{1}{(\sin n z-a)}\left\{1-\frac{i \cos n z}{\left(a^{2}-1\right)^{1 / 2}}\right\}
$$

is regular in $\operatorname{Im}(z) \geqq 0$, and it is possible to proceed initially as in the previous example.

Since $\tilde{\phi}(z)$ has a sequence of simple poles close to the real axis just below it, we choose a contour which avoids the real axis by as wide a margin as possible. It is convenient to use again the three sides of the rectangle of height $L$ having $[-1,1]$ as base. Thus we set

$$
\begin{aligned}
\int_{-1}^{1} w(x) \tilde{c}(x) d x \\
\quad=\operatorname{Re}\left\{\int_{-1}^{-1+i L}+\int_{-1+i L}^{+1+i L}+\int_{+1+i L}^{+1} w(z) \tilde{\phi}(z) d z\right\} .
\end{aligned}
$$

When we come to choose $L$ we encounter a difficulty of a numerical nature. The function $\tilde{\phi}(z)$ has the form

$$
\begin{aligned}
\tilde{\phi}(z) & =-\frac{1}{\left(a^{2}-1\right)^{1 / 2}} \frac{a+\left(a^{2}-1\right)^{1 / 2}-i e^{i n z}}{a+\left(a^{2}-1\right)^{1 / 2}+i e^{i n z}} \\
& =-\frac{1}{\left(a^{2}+1\right)^{1 / 2}}+\frac{1}{\left(a^{2}-1\right)^{1 / 2}} \frac{2 i e^{i n z}}{a+\left(a^{2}-1\right)^{1 / 2}+i e^{i n z}} .
\end{aligned}
$$

Thus for large $\operatorname{Im}(z), \tilde{\phi}(z)$ approaches a constant which overshadows the variations in $\tilde{\phi}(z)$. The calculation involves integrating the sum of this constant and a small varying function around an extensive contour. The contribution of the constant is to add and subtract relatively large numbers in the numerical integration.

Once this particular problem is recognised, a method of avoiding it is easily devised. This is to subtract this constant analytically before carrying out the numerical work. Thus we set

$$
\int_{-1}^{1} w(x) \tilde{c}(x) d x=\frac{1}{\left(a^{2}-1\right)^{1 / 2}} \int_{-1}^{1} w(x) \iota x+\int_{-1}^{1} w(x) c(x) d x
$$

where

$$
c(x)=\tilde{c}(x)+\frac{1}{\left(a^{2}-1\right)^{1 / 2}}
$$

and the corresponding $\phi(z)$ is

$$
\begin{aligned}
\phi(z) & =\tilde{\phi}(z)+\frac{1}{\left(a^{2}-1\right)^{1 / 2}} \\
& =\frac{2 i e^{i n z}}{\left(a^{2}-1\right)^{1 / 2}\left[a+\left(a^{2}-1\right)^{1 / 2}+i e^{i n z}\right]} .
\end{aligned}
$$

The first integral on the right of (5.15) does not involve a critical factor and may be evaluated numerically. The standard procedure may be applied to the second integral leading to a result precisely analogous to (5.6), namely 


$$
\begin{aligned}
& \int_{-1}^{1} \frac{w(x)}{\sin n x-a} d x=\frac{-1}{\left(a^{2}-1\right)^{1 / 2}}\left[\int_{-1}^{1} w(x) d x+\operatorname{Re}\left\{2 \int_{0}^{L} e^{-n y}\right.\right. \\
& \cdot\left[\frac{e^{-i n} w(-1+i y)}{a+\left(a^{2}-1\right)^{1 / 2}+i e^{i n(-1+i y)}}\right. \\
& \left.\quad-\frac{e^{i n} w(1+i y)}{a+\left(a^{2}-1\right)^{1 / 2}+i e^{i n(1+i y)}}\right] d y \\
& \left.\left.+e^{-n L} \int_{-1}^{1} \frac{e^{i n x}}{a+\left(a^{2}-1\right)^{1 / 2}+i e^{i n(x+i L)}} d x\right\}\right] .
\end{aligned}
$$

As before any choice of $L$ is allowable. But if $L$ is chosen to be infinite the final term in (5.18) is zero and the infinite integral may be evaluated using the GaussLaguerre formulas.

This example is interesting because the original critical factor $\tilde{c}(x)$ is one which does not have a Hilbert transform. But after subtracting the constant $-1 /\left(a^{2}-1\right)^{1 / 2}$, the new critical factor $c(x)$ does have a Hilbert transform. $c(x)$ and $\phi(x)$ are among those listed in Table 1.

In a numerical example with $w(x)=(x+2)^{-2}$ and $a=1.1, n=10 \pi$ and the required absolute accuracy $\epsilon=10^{-4}$, we found that the direct evaluation of (5.9) required $3079(\mathrm{ASM})$ or $2049(\mathrm{R})$ real function evaluations. The real integral in (5.18) whose evaluation in this case may be accomplished analytically required $127(\mathrm{ASM})$ or $65(\mathrm{R})$ real function evaluations. The infinite integral $(L=\infty)$ in (5.18) was obtained sufficiently accurately using the ten-point Gauss-Laguerre formula. However, in our calculation the Gauss-Laguerre formulas requiring 4, 6, 8, 10 , and 12 points were evaluated, making a total of 40 complex function evaluations.

6. Concluding Remarks. It is perhaps pertinent to state quite clearly the part played by these types of transformation in the general scheme of computational mathematics. In a very useful article written in 1954, Abramowitz [1] indicates the importance of an initial investigation into some of the analytical properties of a particular quadrature problem before any numerical work takes place. He describes briefly many different transformations which are useful in different problems. These have the effect of reducing significantly the amount of numerical calculation required, and occasionally removing the need for any numerical calculation. The importance of this sort of investigation is emphasised by the fact that this article has been reprinted, thirteen years later, as part of a standard textbook on numerical integration (Davis and Rabinowitz [4]).

The contents of this paper could be considered as an extension of one of the transformations described by Abramowitz. This paper is not directly concerned with quadrature methods per se, that is, expressing an integral as a sum of function evaluations for general use. Rather it is concerned with the prior investigation. As a result one integral which may be difficult to evaluate numerically is replaced by a different integral which may be much easier to evaluate. The work involved in this replacement is analytical in nature. However account has to be taken of the numerical procedures which might be used both in the original calculation and in the transformed calculation in order to see if such a transformation is of practical value. 
Consequently while the mathematical basis of the work presented here is quite rigorous and unambiguous, the application of these results is a question for personal judgement. In the applications we have chosen a particular contour or a particular quadrature rule and have given what we consider adequate reasons for these choices. But we do not claim that these choices which are subjective in nature are necessarily the best, even in our own specific examples. The user should feel free to make different choices according to his particular preferences and his particular problem.

Another aspect of the applications is that different examples require slightly different applications of the same basic theory. For instance in Section 4 we use a different contour from that used in Section 5 . In the second example in Section 5 we subtract out a constant. If there are poles sometimes we take these into account by including them in the 'critical factor' and in other circumstances we use the theory of residues. Thus even in cases where the analytical work involved in evaluating the Hilbert transform has been carried out, some further analytical work (of a more trivial nature) may be required in applying this theory.

However, in spite of the lack of general uniformity, and the restriction to situations which involve functions whose Hilbert transform is known, the examples show that this technique is sometimes very powerful and does cover a wide variety of problems. At the expense of some analytical work, very significant savings in the scope of the numerical part of these calculations can be accomplished.

Argonne National Laboratory

Argonne, Illinois 60439

1. M. Abramowitz, "On the practical evaluation of integrals," J. Soc. Indust. Appl. Math., v. 2,1954 , pp. 20-35. MR 15, 992 .

2. M. Abramowitz \& I. A. Stegun, (Editors), Handbook of Mathematical Functions with Formulas, Graphs, and Mathematical Tables, Nat. Bur. Standards Appl. Math. Ser., 55, Superintendent of Documents, U. S. Government Printing Office, Washington, D. C., 1964; 3rd printing, with corrections, 1965. MR 29 \#4914; MR 31 \#1400.

3. F. L. Bauer, H. Rutishauser \& E. Stiefel, New Aspects in Numerical Quadrature, Proc. Sympos. Appl. Math., vol. 15, Amer. Math. Soc., Providence, R. I., 1963, pp. 199-218. MR 30 \#4384.

4. P. J. Davis \& P. Rabinowitz, Numerical Integration, Blaisdell, Waltham, Mass., 1967. MR $35 \# 2482$.

5. A. Erdélyi, W. Magnus, F. Oberhettinger \& F. G. Tricomi, Tables of Integral Transforms. Vols. I, II, McGraw-Hill, New York, 1954, 1955. MR 15, 868; MR 16, 468.

6. T. HAVIE, "On a modification of Romberg's algorithm," Nordisk Tidskr. InformationsBehandling, v. 6, 1966, pp. 24-30. MR 33 \#3460.

7. J. R. MACDONALD \& M. K. BRACHMAN, "Linear-system integral transform relations," Rev. Mod. Phys., v. 28, 1956, pp. 393-422. MR 18, 652.

8. W. M. MCKEFMAN, "Algorithm 145; adaptive numerical integration by Simpson's rule," Comm. $A C M$, v. 5,1962 , p. 604 . See also: "Certification of algorithm 145; adaptive numerical integration by Simpson's rule," Comm. ACM, v. 6, 1963, pp. 167-168.

9. N. I. Muskhelishvili, Singular Integral Equations, Boundary Problems of Function Theory and Their Application to Mathematical Physics, OGIZ, Moscow, 1946; English transl., Noordhoff, Groningen, 1953. MR 8, 586; MR 15, 434.

10. F. G. Tricomi, Integral Equations, Interscience, New York, 1957. MR 20 \#1177.

11. J. V. USPENSKY, "On the convergence of quadrature formulas related to an infinite interval," Trans. Amer. Math. Soc., v. 30, 1928, pp. 542-559. 